CAE Working Paper \#03-09

\title{
Cantor Type Invariant Distributions in the Theory of Optimal Growth under Uncertainty
}

by

Tapan Mitra

\&

Fabio Privileggi

August 2003 


\title{
Cantor Type Invariant Distributions in the Theory of Optimal Growth under Uncertainty*
}

\author{
Tapan Mitra ${ }^{\dagger}$ and Fabio Privileggi ${ }^{\ddagger}$
}

June 28, 2003

\begin{abstract}
We study a one-sector stochastic optimal growth model, where the utility function is iso-elastic and the production function is of the Cobb-Douglas form. Production is affected by a multiplicative shock taking one of two values. We provide sufficient conditions on the parameters of the model under which the invariant distribution of the stochastic process of optimal output levels is of the Cantor type.

American Mathematical Society Classification Numbers: 26A30, 28A78, 37L40, 60G30, 91B62, 91B70.

Key words: stochastic optimal growth, iterated function system, invariant measure, no overlap property, Cantor function, Lipschitz policy, Hausdorff dimension, singular invariant distribution.
\end{abstract}

${ }^{*}$ This research was undertaken during the second author's visit to the Department of Economics at Cornell University in 2002 as a visiting scholar. A version of this paper was presented at a conference at the University of Paris I in June 2003. Comments received from Kevin Reffett and Manuel Santos at the conference are gratefully acknowledged.

${ }^{\dagger}$ Department of Economics, Cornell University, Uris Hall, Ithaca, NY 14853 (USA); tm19@cornell.edu

${ }^{\ddagger}$ Department of Public Policy and Public Choice, Via Cavour 84, 15100 Alessandria (Italy); fabio.privileggi@unipmn.it 


\section{Introduction}

In the theory of optimal economic growth under uncertainty, relatively little is known about the nature of the stochastic steady-state. Mirman and Zilcha (1975) considered an example with logarithmic utility function and Cobb-Douglas production function (where a multiplicative random shock to production takes one of two values) to show that the invariant distribution of the stochastic process of outputs would be an absolutely continuous function for some chosen parameter values. Montrucchio and Privileggi (1999) considered the same example with different parameter values to show that the invariant distribution of the stochastic process of outputs can be a Cantor function. Mitra, Montrucchio and Privileggi (2002) expanded on this example to establish precise bounds on the parameters of the model under which such Cantor-type and more general singular invariant distributions would arise, as well as bounds on the parameters of the model under which the invariant distribution would be absolutely continuous.

In the above example, it is well-known that the optimal policy function is linear, and it can be explicitly calculated. This allows one to characterize the nature of the stochastic steady state, at least for a wide range of parameter values. However, once one goes beyond this specific example, and allows for instance for the class of iso-elastic utility functions which are not of the logarithmic type, the optimal policy function is necessarily non-linear, and its solution in closed form is not known. Thus, the techniques used in the above example to characterize the nature of the invariant distribution are no longer available, and a more general approach is needed.

In this paper, we consider the case of an iso-elastic utility function, and a Cobb-Douglas production function, and we establish suitable sufficient conditions on the parameters of the model under which the invariant distribution is of the Cantor type. While this special framework is maintained throughout, we believe our approach is applicable in more general settings.

In the first part of the paper, we establish a sufficient condition for the crucial "no-overlap property" of the iterated function system (IFS), generated by the optimal policy function, on the stable invariant set of the stochastic process of optimal output. This property leads to an attractor of the IFS resembling a Cantor set.

We develop further properties of the IFS under suitable restrictions on the parameters. Specifically, we provide conditions under which the maps of the IFS are Lipschitz, with Lipschitz constants which can be directly computed, given the parameters of the model. Using the general theory of IFS, we are then able to identify parameter configurations under which the attractor of the IFS has Lebesgue measure zero, so that the invariant distribution is necessarily singular.

We note, in connection with these results, that some of the mathematical literature on IFS (for non-linear maps) has been developed under the condition that the maps of the IFS are twice continuously differentiable on the relevant state space [see, for example, Matsumoto (1987, 1988)]. In our exercise, the IFS is not a primitive, but rather derived from a stochastic dynamic programming problem. For these problems, such smoothness conditions on the resulting maps are in general not possible to establish. ${ }^{1}$ We have, therefore, not used these results, but have

\footnotetext{
${ }^{1}$ See Araujo (1991) and Santos (1991) for results on the $C^{1}$ differentiability of the optimal policy function, and the difficulties which arise in establishing $C^{2}$ differentiability of the optimal policy function in non-stochastic dynamic programming models. For stochastic dynamic programming models, see Santos and Vigo-Aguiar (1998) for results on the $C^{1}$ differentiability of the optimal policy function. If the random shock has a distribution which is smooth (a condition which is clearly violated in our set-up), then it is possible to show that the optimal policy function is twice continuously differentiable, by using the results of Blume, Easley and O'Hara (1982).
} 
instead based our analysis only on those properties of the value and policy functions, which can be established in our framework.

\section{Preliminaries}

We consider a special case of the standard model of optimal growth under uncertainty as presented in Brock and Mirman (1972), and Mirman and Zilcha (1975). Specifically, the production function is one in which the shocks are multiplicative, so there is a function, $h: \mathbb{R}_{+} \rightarrow \mathbb{R}_{+}$, such that $f(x, r)=r h(x)$ for $(x, r) \in \mathbb{R}_{+} \times S$. The set $S$ of values of the random variable, $r$, is $\{1, q\}$, where $q \in(0,1)$. We interpret the value 1 of $r$ to be the "normal" state, with $q$ representing a downward production shock, occurring with probability $p \in(0,1)$. The function, $h$, is specified to be of the Cobb-Douglas type; that is, there is $\alpha \in(0,1)$, such that $h(x)=x^{1-\alpha} /(1-\alpha)$ for $x \geq 0$. The utility function, $u$, is of the iso-elastic type; that is, there is $\beta \in(0,1)$, such that $u(c)=c^{1-\beta} /(1-\beta)$ for $c \geq 0$. Thus, the "primitives" of our model are the parameters $q, p, \alpha$, $\beta$ and $\delta$, the discount factor, each belonging to $(0,1)$.

One can apply the standard theory of stochastic dynamic programming to obtain an (optimal) value function, $V: \mathbb{R}_{+} \rightarrow \mathbb{R}_{+}$and an (optimal) policy function, $g: \mathbb{R}_{+} \rightarrow \mathbb{R}_{+}$, which we will interpret as the consumption function. That is, given any output level, $y \geq 0$, the optimal consumption out of this output is given by $g(y)$. The optimal input choice (for production in the next period) is then $[y-g(y)]$. We denote $h[y-g(y)]$ by $G(y)$; it is the output obtained in the next period, when $r$ takes the value 1 . Then, $q G(y)$ is the output obtained in the next period, when $r$ takes the value $q$. We will denote the function $q G$ by $H$.

Following Brock and Mirman (1972) and Mirman and Zilcha (1975), one can establish several useful properties of the value and policy functions. We summarize these results (without proofs) in the following Proposition.

Proposition 1 The value function, $V$, and the policy function, g, satisfy the following properties:

(i) $V$ is concave on $\mathbb{R}_{+}$, and continuous on $\mathbb{R}_{++}$;

(ii) $g$ is continuous on $\mathbb{R}_{+}$and $0<g(y)<y$ for $y>0$;

(iii) $g(y)$ and $[y-g(y)]$ are increasing in $y$ on $\mathbb{R}_{+}$;

(iv) $V$ is continuously differentiable on $\mathbb{R}_{++}$, and $V^{\prime}(y)=u^{\prime}[g(y)]$ for $y>0$;

(v) for $y>0$, we have

$$
u^{\prime}[g(y)]=\delta\left\{p V^{\prime}[q G(y)] q h^{\prime}[y-g(y)]+(1-p) V^{\prime}[G(y)] h^{\prime}[y-g(y)]\right\}
$$

(vi) for $y>0$, we have

$$
u^{\prime}[g(y)]=\delta\left\{p u^{\prime}[g(q G(y))] q h^{\prime}[y-g(y)]+(1-p) u^{\prime}[g(G(y))] h^{\prime}[y-g(y)]\right\} .
$$


The optimal policy function leads to the stochastic process:

$$
y_{t+1}=r_{t+1} G\left(y_{t}\right) \quad \text { for } t \geq 0
$$

Alternately, one might say that the optimal policy function leads to an iterated function system (IFS) $\{H, G ; p, 1-p\}$. It is known [from Brock and Mirman (1972)], that there is a unique invariant distribution, $\mu$, of the Markov process described by (3), and the distribution of optimal output at date $t$, call it $\mu_{t}$, converges weakly to $\mu .^{2}$ We are principally interested in the nature of this distribution $\mu$. The distribution function corresponding to $\mu$ is denoted by $F$.

It can be checked that the functions $G$ and $H$ have positive fixed points, and all the fixed points are less than $[1 /(1-\alpha)]^{(1 / \alpha)}$. Denote by $a$ the largest fixed point of $H$, and by $b$ the smallest fixed point of $G$. Following Brock and Mirman (1972), one can establish that $a<b$. The interval $[a, b]$ is an invariant stable set of the stochastic process (3). In particular, the support of $F$ is contained in $[a, b]$. Consequently, in studying the nature of $F$, it is enough to concentrate on the stochastic process (3), with initial output, $y \in[a, b]$. Equivalently, one need only study the iterated function system $\{H, G ; p, 1-p\}$ on the state space $X=[a, b]$.

\section{The No Overlap Property}

Let us examine some elementary features of the iterated function system $\{H, G ; p, 1-p\}$ on the state space $X=[a, b]$. First, we look at the function $H$. We have $H(a)=a$; and, for $y \in(a, b]$, we have $H(y)<y$, so the graph of the map lies below the $45^{0}$ line (except at $a$ ). Further $H(y)$ increases with $y$, reaching $H(b)<G(b)=b$ at $y=b$. Next, we look at the function $G$. Clearly, $G(a)=(a / q)>a$; and for all $y \in[a, b)$, we must have $G(y)>y$, so the graph of the map lies above the $45^{0}$ line (except at $b$ ). Further, $G(y)$ increases with $y$, reaching $G(b)=b$ at $y=b$. The two maps do not overlap if:

$$
H(b)<G(a)
$$

so that the maximum of the $H$ function is less than the minimum of the $G$ function on the state space $X=[a, b]$.

We want to find conditions on the primitives of the model $(q, p, \alpha, \beta$ and $\delta)$ which ensure the no-overlap property (4).

Proposition 2 Suppose the following condition is satisfied:

$$
q^{2 \alpha-1}<[\delta p(1-\alpha)]^{(1-\alpha)}
$$

Then the iterated function system $\{H, G ; p, 1-p\}$ on the state space $X=[a, b]$ has the no overlap property (4).

Proof. Since $H(b) \equiv q G(b)=q b$ and $G(a)=(a / q)$, the no-overlap condition (4) is equivalent to

$$
q^{2}<\frac{a}{b}
$$

We thus need to find a lower bound for $a$ and an upper bound for $b$ such that (6) holds true.

\footnotetext{
${ }^{2}$ For an alternate and simpler approach to this result, see Bhattacharya and Majumdar (2001).
} 
Let us write the stochastic Ramsey-Euler equation (2) [Proposition 1, (vi)] at $y=a$ and at $y=b$ :

$$
\begin{aligned}
\frac{1}{[g(a)]^{\beta}} & =\delta\left\{\frac{p q}{[g(a)]^{\beta}[a-g(a)]^{\alpha}}+\frac{1-p}{[g(a / q)]^{\beta}[a-g(a)]^{\alpha}}\right\} \\
\frac{1}{[g(b)]^{\beta}} & =\delta\left\{\frac{p q}{[g(q b)]^{\beta}[b-g(b)]^{\alpha}}+\frac{1-p}{[g(b)]^{\beta}[b-g(b)]^{\alpha}}\right\}
\end{aligned}
$$

Equation (7) can be re-written as:

$$
\frac{1}{\delta}=\frac{p q}{[a-g(a)]^{\alpha}}+\frac{(1-p)[g(a)]^{\beta}}{[g(a / q)]^{\beta}[a-g(a)]^{\alpha}}
$$

Using the fact that $G(a)=(a / q)$, and so:

$$
[a-g(a)]^{\alpha}=[(1-\alpha) a / q]^{\left(\frac{\alpha}{1-\alpha}\right)}
$$

the equation (9) can be further simplified to read:

$$
\frac{1}{\delta}[(1-\alpha) a / q]^{\left(\frac{\alpha}{1-\alpha}\right)}=q p+\frac{(1-p)[g(a)]^{\beta}}{[g(a / q)]^{\beta}}
$$

The right-hand side expression in (11) is at least as large as $q p$. This yields the inequality:

$$
[(1-\alpha) a / q]^{\left(\frac{\alpha}{1-\alpha}\right)} \geq \delta q p
$$

Manipulating the expression in (12) leads to the following lower bound on $a$ :

$$
a \geq \frac{q(\delta q p)^{\left(\frac{1-\alpha}{\alpha}\right)}}{(1-\alpha)}
$$

Using the upper bound on $b$ :

$$
b \leq[1 /(1-\alpha)]^{(1 / \alpha)}
$$

and (13), we obtain:

$$
q^{(1 / \alpha)}[\delta p(1-\alpha)]^{\left(\frac{1-\alpha}{\alpha}\right)} \leq \frac{a}{b} .
$$

Then, in view of (6), our sufficient condition for no-overlap is:

$$
q^{2}<q^{(1 / \alpha)}[\delta p(1-\alpha)]^{\left(\frac{1-\alpha}{\alpha}\right)},
$$

which can be rewritten as in (5), thus completing the proof.

\section{Remark 1}

(i) The sufficient condition (5) is possibly not the sharpest one can obtain. As is clear from the proof, in certain steps we have used somewhat crude bounds. It would be interesting to attempt a complete characterization of the no-overlap property in terms of the primitives of the model. 
(ii) A necessary condition for (5) to hold is that $(2 \alpha-1)>0$, that is:

$$
\alpha>(1 / 2)
$$

which is the condition for no-overlap in the case when the utility function is logarithmic [see Mitra, Montrucchio and Privileggi (2002)]. Since our sufficient condition does not involve the utility coefficient $\beta$ (and therefore applies to all iso-elastic utility functions) it is to be expected that our condition should turn out to be a stronger restriction than in the logarithmic utility case.

(iii) The sufficient condition (5) is non-vacuous. To see this, note that as $\alpha \rightarrow 1$, the right hand side of (5) converges to 1 , while the left hand side of (5) converges to $q \in(0,1)$. Thus, for $\alpha$ sufficiently close to 1 , condition (5) always holds. That is the no-overlap case arises when the exponent in the Cobb-Douglas production function is "low"; this agrees with the finding in Mitra, Montrucchio and Privileggi (2002) for the logarithmic utility case.

(iv) For a specific numerical case, choose:

$$
q=0.83, \quad p=0.9, \quad \delta=0.9, \quad \alpha=0.95 .
$$

Then the right hand side of (5) can be calculated to be 0.852 , while the left hand side of (5) is 0.846 , and condition (5) holds.

\section{The Lipschitz Property}

We will now show that the iterated function system $\{H, G ; p, 1-p\}$ on $X=[a, b]$ has the Lipschitz property; that is, the maps $H$ and $G$ are Lipschitz continuous on $X$. It is sufficient for this purpose to show that $G$ is Lipschitz continuous.

The Lipschitz continuity of policy functions in non-stochastic models has been studied by Montrucchio (1987). Given the structure of our model, we are able to take a fairly direct approach. The Lipschitz constant that we obtain is possibly not the sharpest possible, because [unlike Montrucchio (1987)] we do not incorporate in it an additional term expressing the degree of concavity of the value function. On the other hand, we can therefore bypass the theory linking the concavity of the value function to the concavity of the utility and production functions. This makes our approach simpler, and the Lipschitz constant is seen to directly depend on the exponents of the utility and production functions.

Our purpose in exhibiting the Lipschitz property of the IFS is to obtain a sufficient condition in terms of the primitives of the model $(q, p, \alpha, \beta$ and $\delta)$. It is, therefore, important to obtain a Lipschitz constant which depends only on these parameters and is independent of the points of evaluations of the derivatives of the utility, production and value functions.

Keeping this objective in mind, we first obtain a positive lower bound, expressed in terms of the parameters $q, p, \alpha, \beta$ and $\delta$, on the optimal propensity to consume, $[g(y) / y]$. This result is clearly also of independent interest as a property of the optimal policy function. 
Proposition 3 Suppose the iterated function system $\{H, G ; p, 1-p\}$ on the state space $X=$ $[a, b]$ has the no overlap property. Further, assume that:

$$
q^{2}>\delta[q p+(1-p)](1-\alpha)
$$

Then, we have the following lower bound on the optimal propensity to consume:

$$
g(y) / y>q^{2}-\delta[q p+(1-p)](1-\alpha) \quad \text { for all } y \in X
$$

Proof. Using (8), and noting that $g(b)>g(q b)$, we have:

$$
\frac{1}{\delta}>\frac{p q}{[b-g(b)]^{\alpha}}+\frac{1-p}{[b-g(b)]^{\alpha}}
$$

On rearranging terms in (17), and denoting $[q p+(1-p)]$ by $\mathbb{E}(r)$ (the expected value of $r$ ),

$$
[b-g(b)]^{\alpha}>\delta \mathbb{E}(r)
$$

By definition of $b$, we have:

$$
b=G(b)=h[b-g(b)]=\frac{[b-g(b)]^{1-\alpha}}{1-\alpha}
$$

This yields:

$$
[b-g(b)]^{\alpha}=[b(1-\alpha)]^{\left(\frac{\alpha}{1-\alpha}\right)}
$$

Combining (18) and (19), we get:

$$
b>\frac{\left[\delta \mathbb{E}_{(}(r)\right]^{\left(\frac{1-\alpha}{\alpha}\right)}}{1-\alpha}
$$

Using (9), and noting that $g(a)<g(a / q)$, we have:

$$
\frac{1}{\delta}<\frac{p q}{[a-g(a)]^{\alpha}}+\frac{(1-p)}{[a-g(a)]^{\alpha}}
$$

On rearranging terms in $(21)$, we get:

$$
[a-g(a)]^{\alpha}<\delta \mathbb{E}(r)
$$

This yields the inequality:

$$
g(a)>a-[\delta \mathbb{E}(r)]^{1 / \alpha}
$$

Thus, for all $y \in X$, using the fact that $g$ is increasing, we obtain from (22):

$$
\frac{g(y)}{y} \geq \frac{g(a)}{b}>\frac{a}{b}-\frac{[\delta \mathbb{E}(r)]^{1 / \alpha}}{b}
$$

Using (20), the second term on the right hand side expression of $(23)$ is less than $(1-\alpha) \delta \mathbb{E}(r)$, while the first term on the right hand side expression of $(23)$ is greater than $q^{2}$, since the nooverlap property holds. Thus, for all $y \in X$,

$$
g(y) / y>q^{2}-(1-\alpha) \delta \mathbb{E}(r)
$$

which establishes the result. 


\section{Remark 2}

(i) In particular, if the sufficient condition (5) on the parameters hold, and (15) holds, then (16) holds, by Proposition 2. In fact, as is clear from the proof, if (5) holds, then this itself ensures that (16) holds. But, this is of interest, of course, only when (15) holds.

(ii) If we let $\alpha \rightarrow 1$, given the other parameters of the model ( $q, p, \alpha, \beta$ and $\delta$ ) fixed, then the sufficient condition (15) is automatically satisfied. In particular, for the numerical example discussed in Remark 1 , with $q=0.83, p=0.9, \delta=0.9, \alpha=0.95$, we have $\delta[q p+(1-p)](1-\alpha)=0.038115$, and $q^{2}=0.6889$, so (5) and (15) are both satisfied. That is, we have the no-overlap property holding, and the propensity to consume has a lower bound of $\left\{q^{2}-\delta[q p+(1-p)](1-\alpha)\right\}=0.650785$.

Proposition 4 Suppose the iterated function system $\{H, G ; p, 1-p\}$ on the state space $X=$ $[a, b]$ has the no overlap property, and (15) holds. Denote $\left\{q^{2}-\delta[q p+(1-p)](1-\alpha)\right\}$ by $m$, and define:

$$
L=\frac{\beta(1-m)}{\delta q p[\beta(1-m)+\alpha m]}
$$

Then, for all $y, z \in X$, we have:

$$
|G(y)-G(z)| \leq L|y-z|
$$

Proof. We will first prove that $G$ is locally Lipschitz on $X$, with Lipschitz constant $L$. That is, we will show that there is some $\varepsilon>0$, such that, whenever $y, z \in X$, and $0<|y-z| \leq \varepsilon$, (25) holds with $L$ defined by (24).

Denote by $m^{\prime}$ the minimum value of $[g(y) / y]$ on $X$; this is well defined by continuity of $[g(y) / y]$ on the (non-empty) compact set $X$. By Proposition 3, we have $m^{\prime}>m$. Now, define

$$
\lambda=\left[1-\frac{\varepsilon}{a-g(a)}\right]^{1+\alpha}
$$

and choose $\varepsilon>0$ sufficiently small so that

$$
\lambda m^{\prime}>m
$$

It is sufficient to show that, with this choice of $\varepsilon$, whenever $y, z \in X$ and $0<z-y \leq \varepsilon$, the inequality (25) holds.

So, let us pick arbitrary $y, z \in X$, with $0<z-y \leq \varepsilon$. Let us write the equation (1) [Proposition $1(\mathrm{v})]$ at $y$ and at $z$ :

$$
\begin{aligned}
& u^{\prime}[g(y)]=\delta\left\{p q V^{\prime}[q G(y)]+(1-p) V^{\prime}[G(y)]\right\} h^{\prime}[y-g(y)] \\
& u^{\prime}[g(z)]=\delta\left\{p q V^{\prime}[q G(z)]+(1-p) V^{\prime}[G(z)]\right\} h^{\prime}[z-g(z)]
\end{aligned}
$$

Thus, subtracting (29) from (28), we obtain:

$$
\begin{aligned}
u^{\prime}[g(y)]-u^{\prime}[g(z)] & =\delta\left\{p q V^{\prime}[q G(y)]+(1-p) V^{\prime}[G(y)]\right\} h^{\prime}[y-g(y)] \\
& -\delta\left\{p q V^{\prime}[q G(z)]+(1-p) V^{\prime}[G(z)]\right\} h^{\prime}[z-g(z)]
\end{aligned}
$$


Since $G(z)>G(y)$, so that $V^{\prime}[q G(z)] \leq V^{\prime}[q G(y)]$ and $V^{\prime}[G(z)] \leq V^{\prime}[G(y)]$, we obtain:

$$
u^{\prime}[g(y)]-u^{\prime}[g(z)] \geq \delta\left\{p q V^{\prime}[q G(y)]+(1-p) V^{\prime}[G(y)]\right\}\left\{h^{\prime}[y-g(y)]-h^{\prime}[z-g(z)]\right\}
$$

We use the Mean Value theorem to obtain $\xi$ satisfying $g(y) \leq \xi \leq g(z)$, such that:

$$
u^{\prime}[g(y)]-u^{\prime}[g(z)]=u^{\prime \prime}(\xi)[g(y)-g(z)]
$$

Similarly, we can find $\zeta$ satisfying $[y-g(y)] \leq \zeta \leq[z-g(z)]$, such that:

$$
h^{\prime}[y-g(y)]-h^{\prime}[z-g(z)]=h^{\prime \prime}(\zeta)\{[y-g(y)]-[z-g(z)]\}
$$

Using (32) and (33) in (31) and changing sign, we obtain:

$$
-u^{\prime \prime}(\xi)[g(z)-g(y)] \geq-\delta\left\{p q V^{\prime}[q G(y)]+(1-p) V^{\prime}[G(y)]\right\} h^{\prime \prime}(\zeta)\{(z-y)-[g(z)-g(y)]\}
$$

Transposing terms, dividing through by $u^{\prime}[g(y)]$, using (28), and rearranging terms, the last inequality becomes:

$$
\frac{g(z)-g(y)}{z-y} \geq-\frac{h^{\prime \prime}(\zeta)}{h^{\prime}[y-g(y)]}\left\{-\frac{u^{\prime \prime}(\xi)}{u^{\prime}[g(y)]}-\frac{h^{\prime \prime}(\zeta)}{h^{\prime}[y-g(y)]}\right\}^{-1}
$$

It remains to convert the right-hand side into terms involving the parameters of our model.

Note that since $\xi$ satisfies $g(y) \leq \xi$, we have:

$$
-u^{\prime \prime}(\xi)=\frac{\beta}{\xi^{1+\beta}} \leq \frac{\beta}{[g(y)]^{1+\beta}}=-u^{\prime \prime}[g(y)]
$$

so that:

$$
-\frac{u^{\prime \prime}(\xi)}{u^{\prime}[g(y)]} \leq\left\{-\frac{u^{\prime \prime}[g(y)] g(y)}{u^{\prime}[g(y)]}\right\} \frac{1}{g(y)}=\frac{\beta}{g(y)}
$$

Since $\zeta$ satisfies $\zeta \leq z-g(z)$, we have:

$$
-h^{\prime \prime}(\zeta)=\frac{\alpha}{\zeta^{1+\alpha}} \geq \frac{\alpha}{[z-g(z)]^{1+\alpha}}=-h^{\prime \prime}[z-g(z)]=-h^{\prime \prime}[y-g(y)]\left[\frac{y-g(y)}{z-g(z)}\right]^{1+\alpha}
$$

Noting that:

$$
\left[\frac{y-g(y)}{z-g(z)}\right]^{1+\alpha} \geq\left[1-\frac{\varepsilon}{a-g(a)}\right]^{1+\alpha}=\lambda
$$

we obtain:

$$
-\frac{h^{\prime \prime}(\zeta)}{h^{\prime}[y-g(y)]} \geq \frac{\alpha \lambda}{y-g(y)}
$$

Using (35) and (36) in (34) and rearranging terms, we get:

$$
\frac{g(z)-g(y)}{z-y} \geq \alpha\left[\frac{\lambda g(y)}{y}\right]\left\{\beta\left[1-\frac{g(y)}{y}\right]+\alpha \frac{\lambda g(y)}{y}\right\}^{-1}
$$


Since $[g(y) / y] \geq m^{\prime}>m$, we have $[\lambda g(y) / y] \geq \lambda m^{\prime}>m$, so $(37)$ implies:

$$
\frac{g(z)-g(y)}{z-y}>\frac{\alpha m}{\beta(1-m)+\alpha m}
$$

By definition of $G$, we have:

$$
\begin{aligned}
\frac{G(z)-G(y)}{(z-y)} & =\frac{[z-g(z)]^{1-\alpha}-[y-g(y)]^{1-\alpha}}{(1-\alpha)(z-y)} \\
& <\frac{[z-g(z)]-[y-g(y)]}{[y-g(y)]^{\alpha}(z-y)} \\
& <\frac{\beta(1-m)}{[y-g(y)]^{\alpha}[\beta(1-m)+\alpha m]}
\end{aligned}
$$

where in the first inequality we used the superdifferentiability property of the concave function $f: \mathbb{R}_{++} \rightarrow \mathbb{R}_{++}$, defined by $f(x)=x^{1-\alpha}$, and the last inequality follows from (38). Since, by Proposition 1 (iii), $[x-g(x)]$ is non-decreasing in $x,(10)$ and (12) imply:

$$
[y-g(y)]^{\alpha} \geq[a-g(a)]^{\alpha} \geq \delta q p
$$

and using this information in (39) yields:

$$
\frac{G(z)-G(y)}{(z-y)}<\frac{\beta(1-m)}{\delta q p[\beta(1-m)+\alpha m]}=L
$$

This establishes that $G$ is locally Lipschitz on $X$, with Lipschitz constant $L$.

It follows from the above result that $G$ is Lipschitz continuous on $X$ with Lipschitz constant $L$. To see this, pick any $y^{\prime}, z^{\prime} \in X$, with $0<z^{\prime}-y^{\prime}$. We can find a positive integer $N$, such that $N \varepsilon \geq\left(z^{\prime}-y^{\prime}\right)$, where $\varepsilon$ was used in the definition of $(26)$. Define $\eta=\left(z^{\prime}-y^{\prime}\right) / N$; then $0<\eta \leq \varepsilon$. We use $\eta$ to define:

$$
\left(y_{0}, y_{1}, \ldots, y_{N}\right)=\left(y^{\prime}, y^{\prime}+\eta, \ldots, y^{\prime}+(N-1) \eta, z^{\prime}\right)
$$

Then, we have, using the fact that $G$ is locally Lipschitz [with the choice of $\varepsilon$ used in the definition of (26)] with Lipschitz constant $L$, and $0<\left(y_{n+1}-y_{n}\right)=\eta \leq \varepsilon$ for $n=0,1, \ldots N-1$,

$$
G\left(z^{\prime}\right)-G\left(y^{\prime}\right)=\sum_{n=0}^{N-1}\left[G\left(y_{n+1}\right)-G\left(y_{n}\right)\right] \leq L \sum_{n=0}^{N-1}\left(y_{n+1}-y_{n}\right)=L\left(z^{\prime}-y^{\prime}\right)
$$

This establishes that $G$ is Lipschitz continuous on $X$, with Lipschitz constant $L$.

Remark 3 Note that the Lipschitz constant L satisfies:

$$
L \leq \frac{1-m}{\delta q p(1-m+\alpha m)} \equiv L^{\prime}
$$

and this gives us a Lipschitz constant $L^{\prime}$ for $G$ that is independent of the parameter $\beta$ of the utility function. 


\section{Cantor Type Invariant Distributions}

Given the results of the previous section, we can apply the standard theory of iterated function systems to observe that:

(i) there is a unique compact set $A \subset[a, b]$, such that $G(A) \cup H(A)=A$; thus, $A$ is a self-similar set;

(ii) $A$ is the support of the unique invariant distribution, $\mu$, of the Markov process, given by (3).

Clearly, the set $A$ is of the Cantor type, and the question arises as to whether this Cantortype set has zero Lebesgue measure. In the following result, we provide a sufficient condition on the parameters of the model under which the set $A$ has zero Lebesgue measure. The sufficient condition used implies in particular that both functions $H$ and $G$ are contractions, but is stronger than this requirement. It seems plausible that the result might be obtained under the weaker sufficient condition that $H$ and $G$ are contractions.

Proposition 5 Suppose the iterated function system $\{H, G ; p, 1-p\}$ satisfies the no-overlap property and the sufficient condition (15). Denoting $\left\{q^{2}-\delta[q p+(1-p)](1-\alpha)\right\}$ by $m$, and $\beta(1-m) / \delta q p[\alpha m+\beta(1-m)]$ by $L$, assume that in addition the following inequality holds:

$$
(1+q) L<1
$$

Then the support $A$ of the unique invariant distribution $\mu$ of (3) is of Lebesgue measure zero, and $\mu$ is singular with respect to Lebesgue measure.

Proof. Define, for the IFS $\{H, G ; p, 1-p\}$,

$$
\begin{aligned}
K(H) & =\min \{K: K \text { is a Lipschitz constant of } H\} \\
K(G) & =\min \{K: K \text { is a Lipschitz constant of } G\}
\end{aligned}
$$

The similarity dimension of $A$ is defined to be (unique) positive root of the equation:

$$
[K(H)]^{d}+[K(G)]^{d}=1
$$

Clearly, $K(G) \leq L$, and $K(H) \leq q L$. Given (40), we have:

$$
K(H)+K(G)<1
$$

Thus, the unique positive root, $\hat{d}$, of (41) must satisfy $\hat{d}<1$. Thus, the similarity dimension of $A$ is less than 1. For a self-similar set, the Hausdorff dimension of the set cannot exceed its similarity dimension [see Yamaguti, Hata and Kigami (1993), Theorem 2.3, p. 20]. Thus, the Hausdorff dimension of $A$ is less than 1.

By definition of Hausdorff dimension, the Hausdorff outer measure of $A$ is zero. Since Lebesgue outer measure coincides with Hausdorff outer measure on $\mathbb{R}$, the Lebesgue outer measure of $A$ is zero. Since $A$ is closed, it is measurable, and hence the Lebesgue measure of $A$ is zero.

Since $A$ is the support of $\mu$, we must have $\mu(X-A)=0$, by definition of support (see, for example, Chung (1974), p. 31). And we have just seen that $\nu(A)=0$, where $\nu$ is Lebesgue measure. Thus, $\mu$ is singular with respect to Lebesgue measure [See Billingsley (1979), p. 374]. 


\section{Remark 4}

(i) The conditions of the Proposition are non-vacuous. If we continue with the numerical example discussed in Remarks 1 and 2, we can check that $L^{\prime}=0.5369037$ (where $L^{\prime}$ is defined in Remark 3), and $(1+q) L^{\prime}=0.9825338<1$, so that $(1+q) L<1$. This holds independent of the value of the parameter $\beta$ of the utility function.

(ii) The formula for $L$ indicates a role of the parameter $\beta$ of the utility function in generating Cantor type invariant distributions with supports of Lebesgue measure zero. Lower values of $\beta$ [that is, utility functions with lower elasticity of marginal utility, $\left.\left\{\left[-u^{\prime \prime}(c)\right] c / u^{\prime}(c)\right\}\right]$ would make the Lipschitz constants of the maps $H$ and $G$ lower, leading more readily to singular invariant distributions being generated. This is a new feature that was not possible to ascertain by studying the example with the logarithmic utility function.

\section{Concluding Remarks}

This paper further develops the work started in Montrucchio and Privileggi (1999), and subsequently investigated more thoroughly in Mitra, Montrucchio and Privileggi (2002) on the nature of the invariant distribution in the standard one-sector optimal growth model under uncertainty.

In the present work, unlike in the previous studies, the optimal policy function cannot be explicitly calculated. So, we develop a more general approach to obtain sufficient conditions on the parameters of the model for the invariant distribution to be a Cantor function.

To obtain such sufficient conditions we rely on the properties of the optimal policy and value functions (Proposition 1). Specifically, a sufficient condition for the no-overlap property of the two maps constituting the IFS (associated with the optimal policy) is given in Proposition 2; this condition is independent of the parameter of the utility function and agrees with the analogous condition, discussed in Mitra, Montrucchio and Privileggi (2002), to get a Cantor attractor. Moreover, under some additional conditions on the parameters, we are able to establish a lower bound on the optimal propensity to consume (Proposition 3), the Lipschitz property of the IFS (Proposition 4) and the singularity of the invariant distribution with respect to Lebesgue measure (Proposition 5).

The approach used in this paper is potentially applicable to the more general setting of Brock and Mirman (1972), where (unlike in the present study) the utility and production functions are not necessarily iso-elastic. By improving the estimate of the largest fixed point of the lower map and the smallest fixed point of the upper map of the IFS, and by establishing a Lipschitz property for the IFS, some general conditions could be obtained under which the invariant distribution of the model is singular. We hope to report on this line of research in the future. 


\section{References}

[1] Araujo, A., The Once but not Twice Differentiability of the Policy Function, Econometrica 59: 1381-1393, 1991.

[2] Bhattacharya, R. and M. Majumdar, On a Class of Stable Random Dynamical Systems: Theory and Applications, Journal of Economic Theory 96: 208-229, 2001.

[3] Billingsley, P., Probability and Measure, New York: John Wiley \& Sons, 1979.

[4] Blume, L., D. Easley and M. O'Hara, Characterization of Optimal Plans for Stochastic Dynamic Programs, Journal of Economic Theory 28: 221-234, 1982.

[5] Brock, W. A. and L. J. Mirman, Optimal Economic Growth and Uncertainty: the Discounted Case, Journal of Economic Theory 4: 479-513, 1972.

[6] Chung, K. L., A course in probability theory, New York: Academic Press, 1974.

[7] Matsumoto, S., Nullity of Measures of Cantor Sets, in Dynamical Systems and Singular Phenomena, edited by G. Ikegami, World Scientific, 1987, 221-223.

[8] Matsumoto, S., Measure of Exceptional Minimal Sets of Codimension One Foliations, in $A$ Fête of Topology:Papers Dedicated to Itiro Tamura, edited by Y. Matsumoto, T. Mizutani and S. Morita, Academic Press, 1988, 81-94.

[9] Mirman, L. J. and I. Zilcha, On Optimal Growth under Uncertainty, Journal of Economic Theory 11: 329-339, 1975.

[10] Mitra, T., L. Montrucchio and F. Privileggi, The Nature of the Steady State in Models of Optimal Growth Under Uncertainty, CAE Working Paper, 2002 (forthcoming in Economic Theory).

[11] Montrucchio, L., Lipschitz Continuous Policy Functions for Strongly Concave Optimization Problems, Journal of Mathematical Economics 16: 259-273, 1987.

[12] Montrucchio, L. and F. Privileggi, Fractal Steady States in Stochastic Optimal Control Models, Annals of Operations Research 88: 183-197, 1999.

[13] Santos, M.S., Smoothness of the Policy Function in Discrete-Time Economic Models, Econometrica 59: 1365-1382, 1991.

[14] Santos, M.S. and J. Vigo-Aguiar, Analysis of a New Dynamic Programming Algorithm Applied to Economic Models, Econometrica 66: 409-426, 1998.

[15] Yamaguti, M., M. Hata and J. Kigami, Mathematics of Fractals, Translations of Mathematical Monographs, Vol. 167, American Mathematical Society, Providence, Rhode Island, 1993. 